\title{
Criptosporidiose: uma revisão sobre a sua implicação na conservação dos mamíferos aquáticos
}

\author{
João Carlos Gomes Borges ${ }^{1,2}$, Leucio Câmara Alves ${ }^{1}$ \& Maria Aparecida da Gloria Faustino ${ }^{1}$
}

Biota Neotropica v7 (n3) - http://www.biotaneotropica.org.br/v7n3/pt/abstract?article +bn01407032007

\author{
Recebido em 16/02/07 \\ Versão reformulada recebida em 22/06/07 \\ Publicado em 06/09/07
}

${ }^{1}$ Laboratório de Doenças Parasitárias dos Animais Domésticos,

Universidade Federal Rural de Pernambuco,

Rua Dom Manoel de Medeiros, s/n, Dois Irmãos CEP 52171-900, Recife, PE, Brasil

e-mail:leucioalves@gmail.com,magfaustino@hotmail.com

${ }^{2}$ Autor para correspondência: João Carlos Gomes Borges, e-mail: jcgborges@hotmail.com

\begin{abstract}
Borges, J.C.G., Alves, L.C. \& Faustino, M.A.G. Cryptosporidiosis: a review about its implication in conservation of aquatic mammals. Biota Neotrop. Sep/Dez 2007 vol. 7, no. 3 http://www.biotaneotropica.org.br/v7n3/pt/abstract?article+bn01407032007. ISSN 1676-0603.

Cryptosporidiosis is an emerging parasitic disease that has been related in several mammal species, including the aquatic ones. This review covers the epidemiology infection by Cryptosporidium spp. in aquatic mammals. The age of the animal, infecting dose and immunological state can influence on the clinical presentation, and when associated with other pathogen, can lead the animal to death. The water supply contamination is water supply is one of the major ways of the pathogen dissemination, which was being identified by different techniques of laboratorial analysis. The adequacy of sanitation structures, the use of appropriate method to inactivate the oocysts and the assurance of personal hygiene norms are some of the recommended way to minimize the dissemination of Cryptosporidium among aquatic mammals.

Keywords: aquatic animals, epidemiology, zoonosis, Cryptosporidium.

\section{Resumo}

Borges, J.C.G., Alves, L.C. \& Faustino, M.A.G. Criptosporidiose: uma revisão sobre a sua implicação na conservação dos mamíferos aquáticos. Biota Neotrop. Sep/Dez 2007 vol. 7, no. 3 http://www.biotaneotropica.org.br/v7n3/pt/abstract?article+bn01407032007. ISSN 1676-0603.

A criptosporidiose é uma doença parasitária emergente que vem sendo relatada em diversas espécies de mamíferos, incluindo os aquáticos. Esta revisão trata da epidemiologia da infecção por Cryptosporidium spp. em mamíferos aquáticos. A idade dos animais, dose infectante e o estado imunológico podem influenciar na apresentação clínica, e quando associados a outros patógenos pode levar o animal a óbito. A contaminação dos recursos hídricos constitui um dos principais meios de disseminação do parasito, que foi identificado por diferentes técnicas de análise. A adequação das estruturas de saneamento, utilização de métodos apropriados para a inativação dos oocistos e garantia das normas de higiene pessoal constituem algumas das maneiras recomendadas para minimizar a disseminação do Cryptosporidium entre os mamíferos aquáticos.
\end{abstract}

Palavras-chave: animais aquáticos, epidemiologia, zoonose, Cryptosporidium. 


\section{Introdução}

São mamíferos aquáticos os animais das Ordens Cetacea e Sirenia, da Subordem Pinnipedia e da Família Mustelidae da Ordem Carnivora, diretamente dependentes do meio aquático para o desenvolvimento de suas atividades vitais, tais como alimentação e/ou reprodução (Brasil 2000). A distribuição destes animais é de extrema abrangência, visto que as espécies marinhas podem ser encontradas em áreas oceânicas (Carwardine 1995, Laist et al. 2001), estuários, baías e foz de rios litorâneos (Flores 1999, Geraci \& Lounsbury 2005, Ibama 2005), enquanto que as fluviais são encontradas na maioria dos principais rios e lagos de águas interiores (Da Silva 1984, Best 1993, Carter \& Rosas 1997, Ibama 2001).

Estas espécies estão sujeitas a diversos fatores que ameaçam as suas populações, dentre estes, o tráfego de embarcações (Kraus 1990, Wells \& Scott 1997), mortalidade acidental em redes de pesca (Parente et al. 2004), atividades petrolíferas (Parente 2005), degradação e contaminação dos ambientes costeiros e de águas interiores (Rosas \& Lehti 1996), caça intencional (Lima 1997, Luna 2001) e a ocorrência de diversos agentes etiológicos, tais como vírus (Rector et al. 2004), bactérias (Vergara-Parente et al. 2003) e protozoários (Dubey et al. 2003). Entre as infecções ocasionadas por protozoários, a criptosporidiose vem sendo considerada como uma doença emergente, oportunista (Xiao et al. 1998) e de distribuição cosmopolita (Current 1983), além de apresentar um caráter zoonótico (Fayer et al. 2004).

Estudos filogenéticos vêm sendo realizados por vários grupos de pesquisadores, porém ainda há divergências quanto à taxonomia do Cryptosporidium. De acordo com Xiao et al. (2004), 13 espécies e alguns tipos de genótipos foram identificados acometendo aves, répteis, peixes (Levine 1984, Alvarez-Pellitero \& Sitja-Bobadilla 2002) e aproximadamente 150 hospedeiros mamíferos, incluindo o homem (Fayer et al. 2000, Xiao et al. 2004).

A ausência de maiores informações sobre espécies de Cryptosporidium que acometem os mamíferos aquáticos dificulta as instituições que mantêm espécimes cativos na adoção de estratégias de manejo que minimizem e/ou eliminem os oocistos deste coccídio, bem como para o melhor entendimento dos aspectos sanitários destes animais de vida livre. Neste sentido, no intuito de propiciar subsídios acerca da ocorrência deste parasito foi elaborado o presente trabalho, o qual teve por objetivo revisar os fatores epidemiológicos relacionados à infecção por Cryptosporidium spp. em mamíferos aquáticos.

\section{Agente Etiológico}

O gênero Cryptosporidium é classificado como um eucarionte pertencente ao Filo Apicomplexa (Levine 1984). Este protozoário foi encontrado pela primeira vez em 1907, em glândulas gástricas de camundongos, sendo denominado Cryptosporidium muris e posteriormente, em 1912, no intestino desta mesma espécie, sendo identificado como C. parvum (Tyzzer 1912). Porém, somente após o primeiro surto acometendo aves domésticas em 1950 (Salvin 1955) e, sobretudo após os relatos em humanos (Laberge et al. 1996) foi que este agente passou a ser pesquisado de maneira mais efetiva.

As espécies de Cryptosporidium são parasitas entéricos e desenvolvem-se no epitélio da mucosa intestinal ou gástrica de diversos vertebrados, diferindo morfologicamente de todos os outros gêneros da subordem Eimeriina, pois são esféricos ou ovóides, medindo de 3 a $8 \mu \mathrm{m}$ de diâmetro e possuem internamente quatro esporozoítos (Xiao et al. 1998).

Entre as espécies do gênero Cryptosporidium descritas, C. hominis, C. muris e C. parvum já foram diagnosticadas em mamíferos aquáticos (Morgan et al. 2000, Deng et al. 2000, Santín et al. 2005).

\section{Ciclo Biológico}

De acordo com as constatações nos diferentes hospedeiros acometidos por Cryptosporidium spp., o ciclo de vida é do tipo monoxeno, com seis estágios de desenvolvimento no organismo hospedeiro: excistação, merogonia, gametogonia, fertilização, formação da parede do oocisto e esporogonia (Gellin \& Soave 1992, Donnelly \& Stentiford 1997, Smith \& Rose 1998).

No transcorrer destes estágios, dois tipos de oocistos são formados, sendo um destes de parede espessa, eliminados na forma infectante através das fezes e resistentes às condições ambientais, sendo responsável pela transmissão do parasito para outros animais; e aqueles de parede delgada, os quais se rompem no hospedeiro e liberam esporozoítos que invadem células epiteliais não infectadas, responsáveis por auto-infecções (Smith 1993, O’Donoghue 1995, Xiao et al. 2004).

A liberação de oocistos presentes nas fezes dos hospedeiros infectados pode acarretar na contaminação de águas superficiais ou reservatórios (Smith 1993, Muller 1999, Machado 2006), os quais têm sido reconhecidos como maiores veiculadores do patógeno pela possibilidade de atingir um imenso contingente de hospedeiros (Laberge et al. 1996), incluindo as diferentes espécies de mamíferos aquáticos.

\section{Ocorrência de Cryptosporidium spp. em Mamíferos Aquáticos}

De acordo com Hughes-Hanks et al. (2005), diversas pesquisas vêm sendo desenvolvidas nos ecossistemas terrestres, porém os estudos e informações de agentes parasitários nos ambientes marinhos ainda são reduzidos. Evidências recentes indicaram a presença do Cryptosporidium spp. infectando uma variedade de organismos aquáticos, entre estes, mariscos (Fayer et al. 2004), mexilhões (Tamburrini \& Pozio 1999) e ostras (Freire-Santos et al. 2000).

O primeiro relato deste coccídio acometendo mamíferos aquáticos foi em dugongo (Dugong dugon), na Austrália, onde se tratava de um espécime em estado de debilidade acentuada, o qual foi eutanasiado e posteriormente submetido a exames "post-mortem" (Hill et al. 1997). Posteriormente foram realizadas análises histológicas deste animal, sendo identificado a presença de Cryptosporidium hominis (Morgan et al. 2000).

Com o despertar para os riscos inerentes à presença deste protozoário acometendo os mamíferos aquáticos, os esforços de investigação foram ampliados em diversos países. Deng et al. (2000) detectaram a presença de $C$. parvum em leões-marinhos da Califórnia (Zalophus californianus), enquanto que Hughes-Hanks et al. (2005) relataram a presença de Cryptosporidium spp. em exemplares da foca-anelada (Phoca hispida), sendo verificada ainda por estes mesmos pesquisadores, a presença deste coccídio acometendo duas espécies de cetáceos, a baleia-da-Groenlândia (Balaena mysticetus) e baleia-franca do norte (Eubalaena glacialis).

Entre os sirênios, além da infecção em dugongo inicialmente descrita, a presença de Cryptosporidium spp. também foi relatada em peixes-boi marinhos mantidos cativos no ambiente natural e em oceanários (Marcondes et al. 2002, Borges et al. 2005), bem como em peixes-boi amazônicos (Borges et al. 2006).

\section{Transmissão}

A criptosporidiose é uma doença que pode ser transmitida pelo contato direto entre pessoas, animais, pessoa-animal ou de forma indireta pelo consumo de água e alimentos contaminados (Rose 1998, Baraldi et al. 1999, Fayer et al. 2004). 
A diversidade de hospedeiros acometidos por Cryptosporidium spp., associado as suas formas de disseminação e a sobrevivência no meio ambiente, contribuíram para a identificação dos oocistos deste coccídio em rios, lagos, estuários e águas oceânicas (Graczyk et al. 1999, Morgan et al. 2000, Fayer et al. 2002, Fayer et al. 2004), o que pode resultar na veiculação entre os mamíferos aquáticos (Deng et al. 2000, Morgan et al. 2000, Olson et al. 2004).

No que concerne à presença de mamíferos aquáticos mantidos em cativeiro, surge a preocupação frente à qualidade da água de piscinas e oceanários, haja visto que em virtude das características dos oocistos, este agente foi encontrado mesmo após tratamento a base de cloro utilizado em alguns parques aquáticos (Causer et al. 2006).

Além disto, a oferta de diferentes itens alimentares, como legumes, verduras, frutas, algas marinhas, fanerógamas e peixes para as diversas espécies de mamíferos aquáticos cativos, podem constituir como fonte de infecção, pois em diversas pesquisas vem sendo relatada a veiculação de oocistos de Cryptosporidium através de tais alimentos, seja decorrente da contaminação em sua origem ou após o processo de manipulação (Monge \& Arias 1996, Ortega et al. 1997).

\section{Sinais Clínicos}

Entre os mamíferos aquáticos, boa parte dos relatos da ocorrência de Cryptosporidium spp. está associada a material coletado de animais em vida livre (Deng et al. 2000, Borges et al. 2006) ou "post-mortem" (Morgan et al. 2000, Hughes-Hanks et al. 2005), o que dificulta a constatação dos sinais clínicos apresentados por estes animais. Porém, de acordo com Hill et al. (1997) a infecção por este coccídio em dugongo, ocasionou sinais de anorexia, acentuada letargia com natação lenta em águas rasas, sendo este animal considerado em estágio terminal. Constatações semelhantes foram relatadas por Borges et al. (2005) ao observarem em um peixe-boi marinho a perda de peso, diarréia, desconforto abdominal e letargia.

Os sinais clínicos presentes em dugongos e peixes-boi marinhos são semelhantes aos transtornos descritos em outros mamíferos terrestres, nos quais foram observados quadros de diarréia aquosa, anorexia, perda de peso, dores abdominais e desidratação (Lallo 1996). Na dependência de fatores como a idade dos animais e o estado imunológico do hospedeiro, a infecção pode variar de subclínica a severa, sendo os animais jovens geralmente mais susceptíveis à infecção com manifestações mais severa da doença (Lallo 1996). Olson et al. (2004) mencionaram a susceptibilidade de focas infectadas pelo coccídio a inanição, predação e/ou a adquirir outros agentes, sendo estas observações pertinentes aos demais mamíferos aquáticos.

Vale ressaltar que, durante estudos envolvendo as populações de peixes-boi marinhos, amazônicos (Borges et al. 2006) e focas-anelada (Santín et al. 2005), nem todos os animais diagnosticados com a infecção por Cryptosporidium spp. possuíam evidências de manifestações clínicas, o que sugere a presença de portadores assintomáticos.

\section{Diagnóstico}

De acordo com Vasquez et al. (1986), não existe unanimidade para recomendar uma técnica como sendo a melhor no diagnóstico da criptosporidiose, porém vale ressaltar que inúmeros métodos vêm sendo utilizados em diversas espécies terrestres, tais como flutuação centrifugada em solução açucarada de Sheather, coloração por safranina azul de metileno (Mundim et al. 1995), auramine-fenol (Casemore et al. 1984), ELISA (Robert et al. 1990), os quais também podem ser empregados em mamíferos aquáticos.

Em estudos direcionados aos hospedeiros aquáticos, amostras fecais de peixes-boi marinhos e amazônicos foram analisadas perante a técnica de sedimentação pelo formol-éter com posterior confecção dos esfregaços, com utilização da coloração de Ziehl-Neelsen modificada (Henriksen \& Pohlenz 1981, Marcondes et al. 2002) e o método de Kinyoun (Brasil 1996). Além destas, a coloração pelo 4'6'-Diamidino-2-Phenylindole (DAPI) foi utilizado para confirmar o diagnostico de Cryptosporidium nos peixes-boi marinhos (Borges et al. 2005).

Entre as pesquisas realizadas em mamíferos aquáticos, a imunofluorescência direta foi a técnica utilizada com a maior frequiência (Olson et al. 1997, Fayer et al. 2005). Contudo, conforme já constatado em pesquisas com mamíferos terrestres, o diagnóstico de criptosporidiose pela microscopia de luz demanda tempo e experiência do investigador para identificar de maneira precisa os oocistos (Lallo \& Bondan 2006, Dall'Olio \& Franco 2004).

Desta forma, métodos de detecção com maior sensibilidade vêm aos poucos sendo empregados, dentre eles a reação em cadeia da polimerase (PCR) conforme a sua utilização em dugongo (Morgan et al. 2000) e golfinho-fliper, Tursiops truncatus (Fayer et al. 2005), sendo salientado a importância desta técnica em pesquisas com mamíferos aquáticos (Hughes-Hanks et al. 2005), pois possibilita detectar seqüências específicas do DNA do parasito (Monis \& Saint 2000, Feng et al. 2001), contribuindo para a elucidação da epidemiologia da criptosporidiose (Hadjusek et al. 2004).

\section{Tratamento}

Até o presente, nenhum protocolo terapêutico foi descrito na utilização de infecções de Cryptosporidium spp. em mamíferos aquáticos. No entanto, os resultados mais expressivos entre os mamíferos terrestres foram alcançados através da utilização do paramomicina e o nitazoxanide, sendo o primeiro um antimicrobiano que reduz a excreção de oocistos (Steiner et al. 1997) enquanto que o segundo tem ação antiprotozoária (Abaza et al. 1998).

Além destes fármacos, a reposição eletrolítica e o suporte nutricional são recomendados como parte do tratamento (Carey et al. 2004), com possibilidades ainda de estratégias terapêuticas promissoras como a utilização do colostro bovino hiperimune anti-C. parvum (Abreu et al. 2003).

\section{Medidas de Prevenção e Controle}

Considerado um coccídio resistente (Carey et al. 2004), os oocistos de Cryptosporidium spp. apresentam características que favorecem a sua rápida dispersão no ambiente, tais como a capacidade de suportar a ação dos desinfetantes comumente utilizados (formoldeído, fenol, etanol, lisol), a possibilidade de atravessar determinados sistemas de filtração de água em decorrência do seu tamanho reduzido, a capacidade de flutuar, a permanência no ambiente durante algumas semanas ou meses e a tolerância em determinadas temperaturas e salinidade (Fayer et al. 2004).

Em virtude da abrangência do ambiente aquático aliado à ampla distribuição das diferentes espécies presentes em águas brasileiras, tornam as medidas de controle do Cryptosporidium limitadas. Sendo assim, para minimizar os riscos inerentes à disseminação da criptosporidiose entre as populações de mamíferos aquáticos de vida livre, torna-se de fundamental importância o controle ambiental, através da adoção de práticas agrícolas para prevenir a poluição de rios por excretas de animais (Graczyk et al. 2000), bem como o incentivo à adequação das estruturas de saneamento, a proteção dos mananciais, educação e orientação sobre descargas de resíduos por embarcações durante atividades náuticas.

No que concerne às medidas de controle dos mamíferos aquáticos cativos, de maneira a minimizar ou eliminar os riscos inerentes à disseminação do coccídio, diversas pesquisas devem ser adotadas. 
A ausência de métodos de tratamento da água em grande parte das instituições onde são mantidos estes animais, bem como às limitações da cloração quando utilizada, aumenta os riscos através da transmissão hídrica. Sendo assim, entre os métodos de inativação recomendados, verifica-se a necessidade de avaliação constante no sistema de filtração, bem como a utilização do ozônio ou a instalação de um sistema ultravioleta nos oceanários e piscinas (Clancy et al. 2000, Kanjo et al. 2000, Causer et al. 2006). Além das medidas citadas, deve-se ainda evitar a presença dos oocistos nos alimentos ofertados e incentivar normas de higiene pessoal entre os tratadores e técnicos envolvidos na manejo dos animais.

\section{Repercussão na Saúde Pública}

A contaminação de oocistos de C. parvum e C. hominis em águas superficiais ou de abastecimento público assume um papel importante no comprometimento ambiental e na saúde pública (Carey et al. 2004), haja visto que inúmeros casos já foram registrados em diferentes países (Fuente et al. 1999, Laubach et al. 2004), inclusive no Brasil (Baraldi et al. 1999, Heller et al. 2004).

Alguns estudos revelaram a diversidade genética do Cryptosporidium spp. e sugerem que a adaptação no hospedeiro e a relação hospedeiro-parasita co-evoluíram contribuindo para a quantidade da heterogeneidade genética deste coccídio (Xiao et al. 2002, Xiao et al. 2004).

Desta maneira, após as constatações de Morgan et al. (2000), que registraram a presença de Cryptosporidium hominis acometendo dugongo, desperta a atenção para a possibilidade destes animais atuarem como reservatórios do coccídio, e segundo Hughes-Hanks et al. (2005) estes achados sugerem uma antropozoonose. De acordo com Buck \& Schroeder (1990), a ocorrência de zoonoses provenientes dos mamíferos aquáticos não é novidade, porém somente nas últimas décadas começou-se a apreciar o potencial para a transmissão de doenças destes animais para o homem.

Antigamente, grande parte das pessoas que contraíam doenças decorrentes da interação com mamíferos aquáticos pertencia a populações isoladas de caçadores, porém atualmente os hospedeiros humanos destas zoonoses, em muitas ocasiões são profissionais ligados a centros de reabilitação ou oceanários, os quais são diariamente expostos ao risco de infecção (Buck \& Schroeder 1990).

Segundo Borges et al. (2006) a presença de Cryptosporidium spp. em peixes-boi amazônicos, representa um risco direto para a possibilidade de disseminação do agente para outros peixes-boi, para as demais espécies que utilizam os recursos hídricos da região e para a população humana, seja em momentos de recreação ou pelo consumo de água contaminada.

\section{Considerações Finais}

As informações sobre a ocorrência de espécies de Cryptosporidium entre mamíferos aquáticos é reduzida, onde no Brasil, este protozoário apenas foi diagnosticado acometendo os peixes-boi marinhos e amazônicos. Desta maneira, surge a necessidade de avaliar a presença deste patógeno em populações de mamíferos aquáticos cativas e de vida livre.

A possibilidade de ocorrência de Cryptosporidium spp. sem manifestações clínicas evidentes reforça a necessidade de monitoramento constante destas populações, através da realização de exames laboratoriais, sobretudo em populações cativas, pois a adoção de medidas de controle do agente favorecerá a reabilitação e manutenção destes espécimes, além de evitar a disseminação dos oocistos para o ambiente através de animais destinados aos programas de reintrodução.

A identificação dos oocistos nos mananciais aquáticos pode ser considerada como um indicador da qualidade ambiental, sendo a presença destes resultante do impacto negativo da infecção humana ou animal para a vida aquática.

No intuito de elucidar fatores epidemiológicos, surge a necessidade de caracterização genética do agente, de maneira a verificar se existe uma especificidade de hospedeiros aquáticos ou trata-se de oocistos do coccídio provenientes de outras espécies terrestres, através das diversas fontes de disseminação, pois somente algumas variantes deste protozoário desencadeiam a infecção e apresentam um potencial zoonótico de transmissão.

As normas para manutenção de mamíferos aquáticos no Brasil são descritas pela Instrução Normativa (IN) $n^{\circ} 3$, de 8 de fevereiro de 2002, elaborada pelo Instituto Brasileiro do Meio Ambiente e dos Recursos Naturais e Renováveis (Ibama), em que se estabelece a necessidade da avaliação dos parâmetros físico-químico e microbiológico da água dos recintos. Diante da possibilidade de veiculação hídrica do Cryptosporidium spp., torna-se oportuno incluir a pesquisa deste coccídio entre as diretrizes recomendadas por esta normativa.

\section{Agradecimentos}

Os autores agradecem ao Programa de Pós-Graduação em Ciência Veterinária, da UFRPE, à FACEPE e a PETROBRÁS, através do patrocínio ao projeto Conservação de Matas Alagadas de Mamirauá.

\section{Referências Bibliográficas}

ABAZA, H., EL-ZAYADI, A.R., KABIL, S.M. \& RISK, H. 1998. Nitazoxanide in the treatment of patients with intestinal protozoan and helminthic infections: a report n 546 patients in Egypt. Curr. Ther. Res. 59(2):116-121.

ABREU, V.J.S., CARDOSO, A.L., PENA, H.F.J., GENNARI, S.M., SINHORINI, I. \& DAMY, S.B. 2003. Avaliação da eficácia do colostro do colostro bovino hiperimune na infecção experimental de roedores com Cryptosporidium parvum. Braz. J. Vet. Res. Anim. Sci. 40:191-198.

ALVAREZ-PELLITERO, P. \& SITJA-BOBADILLA,A. 2002. Cryptosporidium molnarii n. sp. (Apicomplexa: Cryptosporidiidae) infecting two marine fish species, Sparus aurata L. and Dicentrarchus labrax L. Int. J. Parasitol. 32:1007-1021.

BARALDI, S.R., MARQUES, E.G.L. \& DIAS, R.M.D.S. 1999. Ocorrência de Cryptosporidium parvum e Isospora belli na região de Campinas, SP. Rev. Inst. Adolfo Lutz 58(1):97-103.

BEST, R. C. 1993. Inia geoffrensis. Mammalian Species 426:1-8.

BORGES, J.C.G., LIMA, E.C., ALVES, L.C., VERGARA-PARENTE, J.E., FAUSTINO, M.A.G., LIMA, A.M.A. \& LIMA, R.P. 2005. Cryptosporidium spp em peixe-boi marinho (Trichechus manatus manatus) cativo no Centro Mamíferos Aquáticos, Ilha de Itamaracá, no estado de Pernambuco. In I Congresso Nacional de Saúde Pública Veterinária (CNSPV, ed), Guarapari, p. 35.

BORGES, J.C.G., ALVES, L.C., MARMONTEL, M., LIMA, D.S., CASTRO, E.L., VERGARA-PARENTE, J.E., FAUSTINO, M.A.G. \& LIMA, A.M.A. 2006. Infecção por Cryptosporidium spp.: uma ameaça à conservação dos peixes-boi marinhos (Trichechus manatus manatus) e amazônicos (Trichechus inunguis). In VII Congresso Internacional sobre Manejo de Fauna Silvestre na Amazônia e América Latina. (UESC, ed), Ilhéus, p. 29.

BRASIL. 1996. Infecções oportunistas por parasitas em AIDS: técnicas de diagnóstico. Ministério da Saúde. Brasília.

BRASIL. 2000. Portaria n ${ }^{\circ}$ 98, de 14 de abril de 2000. Estabelecimento de normas para a manutenção de mamíferos aquáticos em cativeiro. Diário Oficial da União, Brasília.

BRASIL. 2002. Instrução Normativa ${ }^{\circ}$ 03, de 08 de fevereiro de 2002. Para fins de manutenção em cativeiro das espécies de mamíferos aquáticos. Diário Oficial da União, Brasília.

BUCK, C.D. \& SCHROEDER, J.P. 1990. Public helth significance of marine mammal disease. In CRC Handbook of marine mammal medicine: 
health, disease and rehabilitation, (L. A. Dierauf, ed). Boca Raton CRC Press, p. 163-173.

CAREY, C.M., LEE, H. \& TREVORS, J.T. 2004. Biology, persistente and detection of Cryptosporidium parvum and Cryptosporidium hominis oocyst. Water Res. 38:818-862.

CARTER, S.R. \& ROSAS, F.C. 1997. Biology and conservation of the giant otter, Pteronura brasiliensis. Mammal Rev. 27(1):1-26.

CARWARDINE, M. 1995. Ballenas, delfines y marsopas: guía visual de todos los cetáceos del mundo. In Manuales de identificación (Omega, ed). Barcelona, p.1-56.

CASEMORE, D.P., ARMSTRONG, M., JACKSON, B., NICHOLS, G. \& THOM, B.T. 1984. Screening for Cryptosporidium in stools. Lancet. 1(8379):734-735.

CAUSER, L.M., HANDZEL, T., WELCH, P., CARR, M., CULP, D., LUCHT, R., MUDAHAR, K., ROBINSON, D., NEAVEAR, E., FENTON, S. ROSE, C., CRAIG, L., ARROWOOD, M., WAHLQUIST, S., XIAO, L., LEE, Y.M., MIREL, L., LEVY, D., BEACH, M.J., POQUETTE, G. \& DWORKIN, M.S. 2006. An outbreak of Cryptosporidium hominis infection at an Illiniois recreational waterpark. Epidemiol. Infect. 134:147-156

CLANCY, J.L., BUKHARI, Z., HARGY, T.M., BOLTON, J.R., DUSSERT, B.W. \& MARSHALL, M.M. 2000. Using UV to inactivate Cryptosporidium. J. Am. Water Works Assoc. 92:97-104.

CURRENT, W.L. 1983. Human cryptosporidiosis. New Engl. J. Med. 309:614-615

DALL'OLIO, A.J. \& FRANCO, R.M.B. 2004. Ocorrência de Cryptosporidium spp. em pequenos mamíferos silvestres de três áreas serranas do Sudeste brasileiro. Arq. Bras. Med. Vet. Zoo. 56(1):25-31.

DA SILVA, V.M.F. 1984. Ecologia alimentar dos golfinhos da Amazônia. Dissertação de Mestrado, Universidade do Amazonas, Manaus.

DENG, M., PETERSON, R.P. \& CLIVER, D.O. 2000. First findings of Cryptosporidium and Giardia in California sea lions (Zalophus californianus). J. Parasitol. 86:490-494.

DONNELLY, J.K. \& STENTIFORD, E.I. 1997. The Cryptosporidium problem in water and food supplies. Lebensm. Wiss. Technol. 30:111-120.

DUBEY, J.P., ZARNKE, R., THOMAS, N.J., WONG, S.K., BONN, W.V., BRIGGS, M., DAVIS, J.W., EWING, R., MENSE, M., KWOK, O.C.H., ROMAND, S. \& THULLIEZ, P. 2003. Toxoplasma gondii, Neospora caninum, Sarcocystis neurona, and Sarcocystis canis - like infections in marine mammals. Vet. Parasitol. 116:275-296.

FAYER, R., DUBEY, J.P. \& LINDSAY, D.S. 2004. Zoonotic protozoa: from land to sea. Trends Parasitol. 20(11):531-536.

FAYER, R., MORGAN, U. \& UPTON, S.J. 2000. Epidemiology of Cryptosporidium: transmission, detection, and identification. Int. J. Parasitol. 30:1305-1322.

FAYER, R., SANTÍM, M., DUBEY, J.P., FAIR, P.A., BOSSART, G.D., Sreekumar, C., KWOK, O.C.H. \& THULLIEZ, P.H. 2005. Prevalence of zoonotic protozoo infecting bottlenose dolphins. In Synopsis of Research Meeting Bottlenose Dolphin Health \& Risk (NOAA Techical Memorandum, ed.), South Carolina, p. 56.

FAYER, R., TROUT, J.M., LEWIS, E.J., XIAO, L., LAL, A., JENKINS, M.C. \& GRACZYK, T.K. 2002. Temporal variability of Cryptosporidium in Chesapeake Bay. Parasitol. Res. 88:998-1003.

FENG, X., RICH, S.M., TZIPORI, S. \& WIDMER, G. 2001. Experimental evidence for genetic recombination in the opportunistic pathogen Cryptosporidium parvum. Mol. Biochem. Parasitol. 119:55-62.

FLORES, P.A.C. 1999. Preliminary results of a photoidentification study of the marine tucuxi Sotalia fluviatilis in southern Brazil. Mar. Mammal Sci. 15(3):840-847.

FREIRE-SANTOS, F., LOPEZ, A.M.O., CASTIBLANCO, C.A. V., MAZÁS, E.A., SUÁREZ, E.A. \& MARTÍN, O.G. 2000. Detection of Cryptosporidium oocysts in bivalve mollusses destined for human consumption. J. Parasitolol. 86:853-854.

FUENTE, R. de la., LUZÓN, J.A.H., QUITERIA, R.S., CID, A.G.D., ORDEN, J.A., GARCÍA, S., SANZ, R. \& GÓMES-BAUTISTA, M.
1999. Cryptosporidium and concurrent infections with other major enterophatogens in 1 to 30-day-old diarrheic dairy calves in central Spain. Vet. Parasitol. 80:179-185.

GELLIN, B.J. \& SOAVE, R. 1992. Coccidian infections in AIDS. Toxoplasmosis, cryptosporidiosis and isosporiasis. Med. Clin. N. Am. 76:205-234.

GERACI, J.R. \& LOUNSBURY, V.J. 2005. Marine mammals ashore: a field guide for strandings. 2 ed. National Aquarium in Baltimore, Baltimore.

GRACZYK, T.K., EVANS, B.M., ZIF, C.J., KARREMAN, H.J. \& PATZ, J.A. 2000. Environmental and geographical factores contributing to watershed contamination with Cryptosporidium parvum oocysts. Environ. Res. 82(3):263-271.

GRACZYK, T.C., THOMPSON, R.C.A., FAYER, R., ADAMS, P., MORGAN, U.M. \& LEWIS, E.J. 1999. Giardia duodenalis cysts of genotype a recovered from clams in the Chesapeake Bay subestuary, Rhode River. Am. J. Trop. Med. Hyg. 61:526-529.

HADJUSEK, O., DITRICH, O. \& JAN, S. 2004. Molecular identification of Cryptosporidium spp. in animal and human hosts from the Czech Republic. Vet. Parasitol. 122:183-192.

HELLER, L., BASTOS, R.R.X., VIEIRA, M.B.C.M., BEVILACQUA, P.D., BRITO, L.L.A., MOTA, S.M.M., OLIVEIRA, A.A., MACHADO, P.M., SALVADOR, D.P. \& CARDOSO, A.B. 2004. Oocistos de Cryptosporidium e cistos de Giardia: circulação no ambiente e riscos à saúde humana. Epidemiol. Serv. Saúde. 13(2):79-92.

HENRIKSEN, S.A. \& POHLENZ, J.F.L. 1981. Satining of Cryptosporidium by a modified Ziehl-Neelsen technique. Acta Vet. Scan. 22:594-596.

HILL, B.D., FRASER, I.R. \& PRIOR, H.C. 1997. Cryptosporidium infection in a dugong (Dugong dugon). Aust. Vet. J. 75(9):670-671.

HUGHES-HANKS, J.M., RICKARD, L.G., PANUSKA, C., SAUCIER, J.R., O'HARA, T.M., DEHN, L. \& ROLLAND, M. 2005. Prevalence of Cryptosporidium spp. and Giardia spp. in five marine species. J. Parasitol. 91(5):1255-1228.

IBAMA. 2005. Protocolo de conduta para encalhe de mamíferos aquáticos. Rede de encalhes de mamíferos aquáticos do Nordeste, Recife.

IBAMA. 2001. Mamíferos aquáticos do Brasil: plano de ação. 2 ed., Edições Ibama, Brasília.

KANJO, Y., KIMATA, I., MIYANAGA, S., OKADA, H., BANNO, C., MATSUMOTO, M. \& SHIMADA, Y. 2000. Inactivation of Cryptosporidium spp. oocysts with ozone and ultraviolet irradiation evaluated by in vitro excystation and animal infectivity. Water Sci. Technol. 41:119-125.

KRAUS, S.D. 1990. Rates and potential causes of mortality in north Atlantic right whales (Eubalaena glacialis). Mar. Mammal Sci. 6(4):278-291.

LABERGE, I., GRIFFITHS, M.W. \& GRIFFITHS, M.W. 1996. Prevalence, detection and control of Cryptosporidium parvum in food. Int. J. Food Microbiol. 31:1-26.

LAIST, D.W., KNOWLTON, A.R., MEAD, J.G., COllET, A.S. \& PODESTA, M. Collisions between ships and whales. 2001. Mar. Mammal Sci. 17(1):35-75

LALLO, M.A. 1996. Criptosporidiose canina. Clin. Vet. 1(2):20-22.

LALLO, M.A. \& BONDAN, E.F. 2006. Prevalência de Cryptospordium sp. em cães de instituições da cidade de São Paulo. Rev. Saúde Pub. 40(1):120-125.

LAUBACH, H.E., BENTLEY, C.Z., GINTER, E.L., SPALTER, J.S. \& JENSEN, L.A. 2004. A study of risk factors associated with the prevalence of Cryptosporidium in villages around lake atitlan, Guatemala. Braz. J. Infect. Dis. 8(4):319-323.

LEVINE, N.D. 1984. Taxonomy and review of the cociidian genus Cryptosporidium (Protozoa, Apicomplexa). J. Protozool. 31:94-98.

LIMA, R.P. 1997. Peixe-Boi Marinho (Trichechus manatus): distribuição, status de conservação e aspectos tradicionais ao longo do litoral nordeste do Brasil. Dissertação de Mestrado, Universidade Federal de Pernambuco, Recife.

LUNA, F.O. 2001. Distribuição, status de conservação e aspectos tradicionais do peixe-boi marinho (Trichechus manatus manatus) no litoral norte do 
Brasil. Dissertação de Mestrado, Universidade Federal de Pernambuco, Recife.

MACHADO, E.C.L. 2006. Ocorrência de oocistos de Cryptosporidium spp. em águas superficiais na região metropolitana de Recife/PE. Tese de Doutorado, Universidade Federal de Pernambuco, Recife.

MARCONDES, M.C.C., BRITO, F.L.C., BORGES, J.C.G., LIMA, E.C., ALVES, L.C. \& LIMA,R.P. 2002. Cryptosporidium sp in Antillean manatees (Trichechus manatus manatus) in natural captivity, Paraiba state, Brazil. Florida Marine Mammal Health Conference, Gainesville. Disponível em: http://www.vetmed.utl.edu, (acesso em 29 jul 2002)

MONGE, L. \& ARIAS, M.L. 1996. Presencia de microorganismos patógenos em hortalizas de consumo crudo em Costa Rica. Alan. 46(4):292-294.

MONIS, P.T. \& SAINT, C.P. 2000. Development of a nested-PCR assay for the detection of Cryptosporidium parvum in finished water. Water Res. 35(7):1641-1648.

MORGAN, U.M., XIAO, L., HILL, B.D., O'DONOGHUE, P., JOSEF LIMOR, A.L.A. \& ANDREW THOMPSON, R.C. 2000. Detection of the Cryptosporidium parvum "Human" genotype in a dugong (Dugong dugon). J. Parasitol. 86:1352-1354

MULLER, A.N.B. 1999. Detecção de oocistos de Cryptosporidium spp. em águas de abastecimento superficiais e tratadas da região metropolitana de São Paulo. Dissertação de Mestrado, Universidade de São Paulo, São Paulo.

MUNDIM, M.J.S., SOUZA, L.M., MUNDIM, A.V. \& MORAIS, R.N. 1995. Freqüência de oocistos de Cryptosporidium sp. em fezes de bezerros criados sob condições naturais no município de Uberlândia, analisadas por quatro métodos laboratoriais. Veterinária Noticias. 1(1):33-36.

OLSON, M.E., ROACH, P.D., STABLER, M. \& CHAN, W. 1997. Giardiasis in ringed seals from the western Arctic. J. Wildlife Dis. 33:646-648.

OLSON, M.E., APPELBEE, A. \& MEASURES, L. 2004. Giardia duodenalis and Cryptosporidium parvum infections in pinnipeds. Zoonotic protozoa in the marine environment: a threat to aquatic mammals and public health. Vet. Parasitol. 125:131-135.

O'DONOGHUE, P.J. 1995. Cryptosporidium and cryptosporidiosis in man and animals. Int. J. Parasitol. 25(2):135-139.

ORTEGA, Y.R., ROXAS, C.R., GILMAN, R.H., MILLER, N.J., CABRERA, L., TAQUIRI, C. \& STERLING, C.R. 1997. Isolation of Cryptosporidium parvum and Cyclospora cayetanensis from vegetables collected in markets o fan endemic region in Peru. Am. J. Trop. Med. Hyg. 57(6):683-686.

PARENTE, C.L. 2005. Monitoramento de mamíferos marinhos durante estudos sísmicos no nordeste do Brasil. Dissertação de mestrado, Universidade Federal de Pernambuco, Recife.

PARENTE, C.L., VERGARA-PARENTE, J.E. \& LIMA, R.P. 2004. Strandings of Antillean manatees, Trichechus manatus mantus, in northeastern Brazil. Lajam. 3(1):69-75.

RECTOR, A., BOSSART, G.D., GHIM, S.J., SUNDBERG, J.P., JENSON, A.B. \& RANST, M.V. 2004. Characterization of a novel close-to-root papillomavirus from a Florida manatee by using multiply primed rolling-circle amplification Trichechus manatus manatus papillomavirus type 1. J. Virol. 78(22):12698-12702.

ROBERT, B., GINTER, A., COLLARD, A. \& COPPE, P. 1990. Diagnosis of bovine cryptosporidiosis by enzyme-linked immunosorbent assay. Vet. Parasitol. 37:1-8.

ROSAS, F.C.W. \& LEHTI, K.K. 1996. Nutritional and mercury content of milk of the Amazon River e Dolphin, Inia geoffrensis. Comp. Biochem. Physiol. 115:117-119.

ROSE, J.B. 1998. Ocurrence and significance of Cryptosporidium in water. J. Am. Water Works Assoc. 80:53-58.

SALVIN, D. 1955. Cryptosporidium spp. meleagridis (sp. nov). J. Comp. Pathol. 65:262-266.

SANTÍN, M., DIXON, B.R. \& FAYER, R. 2005. Genetic characterization of Cryptosporidium isolates from ringed seals (Phoca hispida) in Northern Québec, Canada. J. Parasitol. 91(3):712-716.

SMITH, J.L. 1993. Cryptosporidium spp. and Giardia as agents of foodborne disease. J. Food Protect. 56(5):451-461.

SMITH, H.V. \& ROSE, J.B. 1998. Waterborne Cryptosporidiosis: current status. Parasitol. Today 14:14-22.

STEINER, T.S., THIELMAN, N.M. \& GUERRANT, R.L. 1997. Protozoal agents: what are the dangers for the public water supply? Annu. Rev. Med. 48:329-340.

TAMBURRINI, A. \& POZIO, E. 1999. Long term survival of Cryptosporidium oocysts in seawater and in experimentally infected mussels (Mytilus galloprovincialis). Int. J. Parasitol. 29:711-715.

TYZZER, E.E. 1912. Cryptosporidium parvum (sp. nov), a coccidium found in the small intestine of the common mouse. Arch. Protistenkd. 26:394-412.

VASQUEZ, I.H.G., RESTREPO, M.I. \& BOTERO, D. 1986. Cryptosporidiosis. Biomédica 6(12):48-70.

VERGARA-PARENTE, J.E., SIDRIM, J.J.C., TEIXEIRA, M.F.S., MARCONDES, M.C.C. \& ROCHA, F.G. 2003. Salmonellosis in an Antillean manatee (Trichechus manatus manatus) calf: a fatal case. Aquat. Mammals 29(1):131-136.

WELLS, R.S. \& SCOTT, M.D. 1997. Seasonal incidence of boat strikes on bottlenose dolphins near Sarasota, Florida. Mar. Mammal Sci. 13(3):475-480.

XIAO, L., FAYER, R., RYAN, U. \& UPTON, S.J. 2004. Cryptosporidium taxonomy: recent advances and implications for public health. Clin. Microbiol. Rev. 17(1):72-97.

XIAO, L., SULAIMAN, I., FAYER, R. \& LAL, A.A. 1998. Species and strainspecific typing of Cryptosporidium parasites in clinical and environmental samples. Mem. I. Oswaldo Cruz 93(5):687-692.

XIAO, L., SULAIMAN, I.M., RYAN, U.M., ZHOU, L., ATWILL, E.R., TISCHLER, M.L., ZHANG, X., FAYER, R. \& LAL, A.A. 2002. Host adaptation and host-parasite co-evolution in Cryptosporidium: implications for taxonomy and public health. Int. J. Parasitol. 32:1773-1785. 